\title{
Special issue editorial: Virtual Agents for Social Skills Training
}

\author{
Merijn Bruijnes $^{1} \cdot$ Jeroen Linssen ${ }^{2} \cdot$ Dirk Heylen $^{1}$
}

Published online: 14 January 2019

(c) Springer Nature Switzerland AG 2019

The topic of this special issue of the Journal on Multimodal User Interfaces is Virtual Agents for Social Skills Training. In this issue four papers investigate how interactive, multimodal technology such as virtual agents can be used in social skills training.

In recent years, intelligent virtual agents (IVAs) have been used in many settings, for example, for education, guidance, and as assistants in domains ranging from museum facilities to healthcare [4]. Studies revolving around these agents can be categorized in three research topics, as Norouzi et al. [1] propose: non-verbal and verbal behaviour, physical appearance and identities, and applications. Based on their review of 247 papers on IVAs in the past 15 years, they highlight three underrepresented research topics: virtual and augmented reality, human collaboration with agents, and multimodal interfaces. In this special issue on Virtual Agents for Social Skills Training of the Journal on Multimodal User Interfaces, we present a selection of studies addressing these research topics.

Veltman, de Weerd, and Verbrugge in their paper on Training the use of theory of mind using artificial agents argue that when interacting with virtual agents, high fidelity of the training situation at hand may have adverse effects, e.g., graphics may distract users or raise undesired expectations of the virtual agents' affordances, and make it increasingly difficult to attribute found effects to their actual causes. Veltman et al. [7] work looks into a low-fidelity simulation which is aimed at training users' higher-order Theory of Mind (ToM) thinking. Through a game in which players need to reason about each other's thoughts, this higher order thinking is beneficial to winning. By extension, ToM reasoning is useful for a variety of social skills, especially for children with autism spectrum

Merijn Bruijnes

m.bruijnes@utwente.nl

Jeroen Linssen

j.m.linssen@saxion.nl

1 Human Media Interaction, University of Twente, Enschede, Netherlands

2 Ambient Intelligence, Saxion University of Applied Science, Enschede, Netherlands disorder. An experiment carried out by Veltman et al. goes on to show that, given information on the level of ToM a virtual agent used, participants would fare well, winning from them after several rounds of playing. This way, participants even adopted fourth-order ToM reasoning. However, when the virtual agents switched strategies (reasoning on a different level every round), participants could rarely win. We agree with the authors that a valuable next step of this line of research is extending this training approach to other settings. We concur with the reviewers in suggesting to increase the fidelity of the game and the agents, embodying them, and investigating to what extent nonverbal cues of the agents, and less explicit cues on the level of ToM adopted by agents, can influence learning effects in participants.

Recht and Grynszpan in their paper The sense of social agency in gaze leading compare low- and high-fidelity approaches to social cues is that of Recht and Grynszpan [5]. In their study, they evaluated the effects of a simple graphic arrow (non-socially familiar feedback) and a realistic face of a virtual human (socially familiar feedback) in the attribution of agency. Participants rated the degree to which the avatar and arrow were controlled by their gaze. This small, compartmentalized task allows for the evaluation of the very specific task of deictic gaze cueing. By measuring the response times of participants in following the direction of either the agents' gaze or the arrow's point, the authors show that the latter are shorter. This may be related to the notion that a social agent is more keenly observed by participants, and attributed a higher degree of agency. A next step, which both the authors and the reviewers agree upon, is the evaluation of human versus computational control over the avatar, and how that would influence the attribution of social agency. Additionally, this opens the way for research into embedding the task of social gaze leading in broader, more expansive interactions, e.g., by tying it to research on how personalities influence the nonverbal behaviours of virtual agents.

Sveinbjörnsdóttir, Jóhannsson, Oddsdóttir, Sigurðardóttir, Valdimarsson, and Vilhjálmsson in their Virtual discrete trial training for teacher trainees paper investigate the training of Discrete Trial Training (DTT). This method is used 
by teachers to teach children on the autism spectrum certain skills. These teachers require training, but training is only possible when people to practice on are available. Virtual reality (VR) could address this by having teacher practice DTT skills on virtual agents. This paper describes a study aimed to assess the efficacy of a DDT training for teachers using virtual reality. The steps of DTT are taught to the participants via a lecture and/or a practice session with a virtual agent. In a single subject design four participants were assessed on the correctness of implementing these steps. The performance of the participants increased after the lecture and after the VR-training showing that teachers can learn DDT in VR [6]. We agree with the reviewers that four participants is a low number of for an evaluation study. However, all participants are autistic children's therapists adding to the validity of the findings. The use of end-users, rather than a convenience sample of university students, should be applauded. Yet, to generalise the results presented in this paper more participants (or studies) are needed.

Ochs, Mestre, de Montcheuil, Pergandi, Saubesty, Lombardo, Francon, and Blache in their Training doctors' social skills to break bad news: evaluation of the impact of virtual environment displays on the sense of presence paper study three different type of virtual environments: PC screen, head mounted display, and virtual reality cave. These environments are compared in a setting where doctors have to train their social skills in bad news conversations. Their systems are evaluated on user experience and the perception of presence. A within subject design experiment with 22 participants of which 10 real medical doctors was executed. The users had to communicate a medical situation to a virtual patient via the three different displays. Results showed higher scores on the sense of presence for the HMD and virtual reality cave compared to the PC screen [2]. This adds to the growing body of evidence that more immersive devices lead to higher presence in the virtual world. Additionally, experts (doctors) tend to more involved in the interaction compared to naïve participants and this effect is strongest for the virtual reality cave condition. Future work should investigate whether this effect is generalisable.

Finally, we would like to give some general recommendations to researchers interested in Virtual Agents for Social Skills Training (VASST). Training applications cannot be viewed separately from the training environment or the curriculum in which they will be used. One observation is that training actors can feel threatened by this emerging technology. However, we argue that VASST technology will be an addition to existing training methods and not a replacement. In any case, it means that the creators of VASSTs should collaborate with the educational and domain experts (and other stakeholders) that will utilize the technology or be affected by its implementation. We argue that the collaboration should start as early as possible in a participatory design approach to ensure the right things are taught and the teaching makes sense in the rest of the curriculum. An added benefit is that it will be clear on what task-related facets the VASST should be evaluated: can it teach the student for which the system is intended the correct knowledge that should be learned. Besides a task-related evaluation, a 'social-related' evaluation makes sense. After all, a virtual agent is chosen as the interface method for the reason that it can imitate a social interaction. One effort that deserves attention is the Open Science Foundation workgroup on Evaluation Instruments [3]. A workgroup that aims at creating a validated standardised questionnaire instrument to evaluate human interaction with a social agent. This instrument will help researchers to make claims about people's perceptions, attitude and beliefs towards their agent. It will allow agents to be compared across user studies, and importantly, it helps in replicating our scientific findings. This is essential for the community if we want to make valid claims about the impact that our social agents can have in domains such as health, entertainment, and education.

\section{References}

1. Norouzi N, Kim K, Hochreiter J, Lee M, Daher S, Bruder G, Welch G (2018) A Systematic survey of 15 years of user studies published in the intelligent virtual agents conference. In: Proceedings of the 18th international conference on intelligent virtual agents, pp 17-22

2. Ochs M, Mestre D, de Montcheuil G, Pergandi JM, Saubesty J, Lombardo E, Francon D, Blache P (2019) Training doctors' social skills to break bad news: evaluation of the impact of virtual environment displays on the sense of presence. J Multimodal User Interfaces. https://doi.org/10.1007/s12193-018-0289-8

3. Open Science Foundation Workgroup on Evaluation Instruments (2018). https://osf.io/6duf7/

4. Provoost S, Lau HM, Ruwaard J, Riper H (2017) Embodied conversational agents in clinical psychology: a scoping review. J Med Internet Res 19(5):e151. https://doi.org/10.2196/jmir.6553

5. Recht S, Grynszpan O (2019) The sense of social agency in gaze leading. J Multimodal User Interfaces. https://doi.org/10.1007/ s12193-018-0286-y

6. Sveinbjörnsdóttir B, Jóhannsson SH, Oddsdóttir J, Sigurðardóttir TP, Valdimarsson GI, Vilhjálmsson HH (2019) Virtual discrete trial training for teacher trainees. J Multimodal User Interfaces. https:// doi.org/10.1007/s12193-018-0288-9

7. Veltman K, de Weerd H, Verbrugge R (2019) Training the use of theory of mind using artificial agents. J Multimodal User Interfaces. https://doi.org/10.1007/s12193-018-0287-x

Publisher's Note Springer Nature remains neutral with regard to jurisdictional claims in published maps and institutional affiliations. 